DEPAR'TAMWNTOO DE INDÚSTRIA, INSPEÇAO E CONSERVAÇĀO DF PRODU'TOS ALIMENTICIOS DE ORIGEM ANIMAL

Diretor: Prof. Paschoal Mucciolo

DIVISION OF BACITRIOLOGY, AMERICAN MEAT INSTITUTE FOUNDATION, UNIVERSITY OF CHICAGO, ILIINOIS

Director: Dr. C. F. Niven, Jr.

\title{
EFFECT OF BIOTIN ANTAGONISTS AND RELATED SUBSTANCES UPON THE GROWTH OF THE COAGULASE-NEGATIVE STAPHYLOCOCCI $\left(^{*}\right)$
}

\author{
P. Mucciolo $\left.{ }^{* *}\right)$ \\ C. F. Niven, JR.
}

One of the most important public health problems confronting the food industry today is hacterial food poisoning caused by certain members of the genus Staphylococcus. Under favorable conditions these organisms may elaborate in a food a toxic substance known as "enterotoxin". Foods containing sufficient quantities of enterotoxin when consumed may produce the commonly encountered synıtoms of acute prostration, diarrhea, and vomiting. Although rarely fatal, this type of food poisoning attracts a great deal of public attention, especially when large numbers of persons are affected at one occasion.

The laboratory investigations of food poisoning outbreaks have a number of limitations, and a great deal of the information concerning of the responsible food item in a particular food poisoning outbreak is dependent upon epidemiological studies rather than laboratory examination of the foods consumed. The reasons for these limitations are many, including the fact that no adequate laboratory test exists that will differentiate an enterotoxigenic Staphylococcus strain froni one that produces no toxin.

In recent years work by Evans (1948), Evans and NIVEN (1950), and Evans, Buettner and Niven (1950) has definitely indicated that all enterotoxigenic Staphylococcus strains are confined to one homogeneous group, namiely, the coagulase-positive strains. Not all coagulase-positive staphylococci are capable of producing the toxin, as detected by rhesus monkey feeding tests, but from

$\left({ }^{*}\right)$ Presented at the First Pan American Congress of Veterinary Medicine, l'eru Limia, October 20 to 28, 1951.

(**) Recipient of a Rockefeller Foundation fellowship. 
the results of Evans et al., it would appear that approximately 75 per cent of such strains are enterotoxigenic according to the techniques employed.

It is known that a great deal of growth of a food-poisoning Staphylococcus, thus resulting in a very large population, must take place in a food item before sufficient enterotoxin is produced to cause illness. It then appears that a help. ful laboratory test to be performed upon suspected foods would be the quantitative determination of the numbers of coagulase-positive staphylococci present in the food. At the moment, however, no such laboratory method is available to obtain this information. At best, one can only determine by very crude methods the approximate numbers of such microorganisms in the sample examined. The reason for this dilema is the fact that other saprophytic staphylococci are invariably encountered in foods that have physiological characteristics similar to the coagulase-positive group, thus preventing a differentiation directly on the plating medium, and making it necessary to isolate individual colonies for positive identification.

In attempts to develop differential of selective plating media for the quantitative detection of coagulase-positive staphylococci, many techniques have been employed, most of which have relied mainly upon some outstanding characteristic that is possessed by this group of bacteria, such as mannitol or lactose fermentation, blood hemolysis, gelatin liquefaction, or tolerance to various chemicals or unfavorable environmental conditions. Since these attempts have failed, other peculiarities of the coagulase-positive staphylococci have been sought.

Recently, Gretler (1950), and Gretler and Evans (1951) have reported a remarcable difference existing between the coagulase-positive staphylococci and the closely related saprophytes with respect to their biotin requirements. Of the 90 coagulase-positive strains tested none required biotin for growth in a simplified casein hydrolysate medium. On the other hand, of the 4.6 coagulasenegative strains tested 38 required biotin for growth while the remaining 8 strains were greatly stimulated by this vitamin. Virtually all of these coagulase. negative strains had been isolated from foods, some of which had been strongly implicated by circumstantial evidence as the cause of food poisoning outbreaks. By animal feeding tests none of these coagulase-negative staphylococci appeared to be able to produce enterotoxin.

It would then seem possible to devise a plating, or liquid medium, containing an anti-biotiri substance in which the growth of the coagulase-negative staphylococci would be inhibited, but yet would have no effect upon the growth of the coagulase-positive group. This medium would then allow a clear-cut method of quantitatively enumerating the coagulase-positive staphylococci without inter- 
ference from the saprophytic strains. This investigation deals with the biotin nutrition of the coagulase-negative staphylococci with the above-stated aim in mind.

\section{HXX I'HRIMLNTAL}

Cultures: All of the cultures used in this study were obtained from the collection of the Division of Bacteriology, American Meat Institute Foundation. These cultures hat been isolated from foods suspected of having caused food poisoning outbreaks, but no evidence of their enterotoxigenicity has been obtained by animal feeding tests. The physiological characteristics of most of these cultures have been reported by Evans and Niven (1950).

Techniques: Throughout most of the work the cultures were carried by serial transfer in a trypticase soy broth (BBL) supplemented with 1 gram of sodium cilrate and 0.2 grams of cystine per liter. In order to prepare an inoculum for the simplified media used in the experiments, a 24-hour culture of the organism was centrifuged, washed once with water and then diluted 1:100 in sterile distilled water. One drop of this diluted suspension was used as an inoculum for each of the tubes in the various experiments.

The biotin-free basal medium used throughout this study is listed in Table 1. This medium was identical with that employed by GRETLER (1950) in his studies on the nutrition of these staphylococci. For each of the experiments the medium was prepared from stock solutions of the various constituents that were preserved with 1 per cent of a volatile preservalive solution deseribed by HUTNER and BJERKNEs (1948). All of the stock solutions were stored in the refrigerator.

All glassware used in these studies was thoroughly washed, soaked in a dichromate cleaning solution overnight, thoroughly rinsed with tap and deionized water, and then dried. The tubes of medium were capped with aluminum caps especially designed for that purpose. These caps were routinely cleaned by boiling in deionized water, followed by a thorough rinse in deionized water.

Most of the studies were conducted with the' use of $18 \mathrm{~mm}$. pyrex test tubes especially calibrated as to diameter, and candled to climinate serious errors with respect to optical density.

Double strength basal medium was added to the tubes in $5 \mathrm{ml}$. quantities and various increments of the test substances added along with distilled water to bring the final volume to $10 \mathrm{ml}$. Before inoculating, the tubes were autoclaved for 15 minutes at $121^{\circ} \mathrm{C}$. 
The tubes were incubated at $30^{\circ} \mathrm{C}$. and growth was quantitatively determined at the specified interval of time. Growth was estimated turbidimetrically with aid of a Coleman Spectrophotometer, mode\} II, set at $660 \mathrm{~m} \mu$. and equipped with a PC-5 filler. An uninoculated tube of medium was used as a reference to give an optical density of zero. Readings were recorded in terms of optical density multiplied by 100 in order to dismiss with decimals.

\section{RHSUL'TS}

The substances tested throughout this study were avidin, homobiotin, desthiobiotin and biocytin. Results ohtained with each of these substances will be discussed in turn.

Avidin: - This substance obtained from egg white is said to be a basic protein which combines stoichiometrically with biotin, thus depriving the test microorganism or the animal of this essential vitamin. Thus, in a basal medium having a constant concentration of biotin, increasing increments of avidin would be expected to inversely affect growth of the test organism resulting a straight line effect. This effect has been demonstrated with many hiotin-requiring microorganisms.

On the other hand, GRETLER (1950) reported that a stoichionetric relationship did not exist between avidin and biotin when tested with a biotin-requiring, coagulase-negative Staphylococcus, strain H 17C. Growth of this microorganism appeared to be constant up to a critical level of avidin, beyond which growth abruptly ceased. If still higher concentrations of avidin were added to the me. dium, growth again resulted; the amount directly depended upon the concentration of avidin present.

In the present study attempts were made to confirm GRETLFR's observations. Results of a typical experiment are given in Table 2. The basal medium contained just sufficient biotin $(2 \mathrm{~m} \mu \mathrm{g} . / 10 \mathrm{ml}$.) to allow maximum growth. Since pure avidin was not available, a bacteriologically filtered egg white preparation was used as a crude source of this substance. Dilutions of the sterile raw egg white were added aseptically to the previously sterilized basal medium.

As shown in Table 2, the phenomenon of GreTLER was confirmed. Crowth of the test organism was unaffected up to a level of 0.15 per cent. Just beyond this concentration growth of the test organism was greatly reduced. However, if still higher levels of egg white were added, growth again increased up to approximately that occurring in the control tube. 
In the presence of 20 millimicrograms of biotin per $10 \mathrm{ml}$. of medium egg white did not affect growth in any concentration tested. The reason for the lack of a stoichiometric relationship between avidin and biotin among the coagulasenegative staphylococci is unknown. Obviously, it would be desirable to repeat these experiments using purified avidin preparations. Crowth in the presence of very high levels of egg white as shown in Table 2 is believed to be due to the presence of "biotin-sparing" substances in the egg white that are unaffected by avidin.

Although of great interest, these results would indicate that avidin would have little value in the development of a plating medium for the quantitative dectetion of coagulase-positive staphylococci.

Desthiobiotin: - Structurally, desthiobiotin is similar to biotin with the exception of the removal of the sulfur atom, thus opening the cyclic strueture of that part of the molecule. It has been variously reported as a biotin active substance for some test microorganism, as well as having anti-biotin properties for other test organisms (Stockes and Gunness, 1945; Rubin, Drekter and MAYER, 1945).

In the present experiments desthiobiotin was found to replace biotin for 17 of the 18 roagulase-negative staphylococci tested. The results are recorded in Table 3. For culture S19 desthiobiotin appeared to have weak anti-biotin properties. No growth occurred during the first three days' incubation in those tubes containing this substance but further incubation resulted in growth in all tubes. Although the exact molar inhibitory ratio between desthiobiotin and biotin was not determined for this microorganism, it would be expected to be very high. As shown in Table 3,2 millimicrograms of biotin completely reversed the growth inhibition of 200 micrograms of desthiobiotin.

Subsequent experiments indicated that the concentration of desthiobiotin added to the medium in the above-cited experiments was far higher than was necessary. For example, in comparison to 0.04 millimicrograms of biotin per $10 \mathrm{ml}$. being necessary for half-maximum growth for culture $\mathrm{H} 3 \mathrm{~A}$, it was found that 0.1 millimicrogram of desthiobiotin would afford the same amount of growth. A similar concentration of desthiobiotin was found to be necessary for halfmaximum growth for culture H2C. These quantitative estimations were conducted under aerobic conditions ( $10 \mathrm{ml}$. of medium in $125 \mathrm{ml}$. erlenmeyer flasks) since quantitative growth response was difficult to achieve for many of the microorganisins in the deep culture test tubes ordinarily employed. The experine?ts 
with desthiobiotin were further complicated by the apparent spontaneous decomposition of this substance when held in aqueous solutions.

Homobiotin: - This substance is a chemical homolog of biotin containing 6 carbons in the aliphatic side chain rather than the 5 carbons found in the biotin molecule. Homobiotir has been reported by GoLDisRg et al. (1947), and Rubin and Scheiner (1949), to have very potent anti-biotin properiles for certain yeast and Lactobacillus strains. On the other hand, Pia.chlik and l.achstein (194.9) reported that this substance may replace biotin for other yeast itrains.

In the present study 17 of the 18 Staphylococcus strains studied were able to use homobiotin as a biotin source. One strain (\$36) was found to be strongly inhibited by this substance. In contrast to desthiobiotin for strain S19, 2 milli. micrograms of hiotin failed to counteract the anti-biotin properties of 200 micrograms of homobiotin for this culture, thus indicating a low molar inhibition ratio between the two substances. (The molar inhibition ratio is defined as the moles of antibiotin required to imhibit one mole of biotin.)

Table 4 lists the growth response obtained with strain $\mathrm{H17C}$ when increasing increments of homohiotin were added to the basal nedium. As was usually the case, a moderate amount of growth of this strain usually occurred in the basal medium alone, thus indicating a small contamination of biotin in the medium or a carrvover of this growth factor in the inoculum. However, small quantities of homobiotin completely inhibited growth. On the other hand, larger quantities of homobiotin afforded growth that equalled maximum growth obtained with biotin. No explanation can be offered for this apparent antibiotin property of homobiotin in small concentrations in contrast to its growth stimulation in higher concentrations. As shown in Table 4, the inhibitory effect of this substance could not be demonstrated in any concentration tested in the presence of 2 millimicrograms of biotin per $10 \mathrm{ml}$. of medium. The experiment was repeated with only 0.5 millimicrogram of biotin added per $10 \mathrm{ml}$. of medium. Even at this concentration no antibiotin effect of homobiotin could be detected in any of the levels tested.

The question arises as to whether homobiotin is being used as such by these microorganisms or is first being converted into biotin. BEI.CHER and Lichstein (1949) ohtained rather definite evidence that the homolog was not being converted into biotin before being used by certain yeast strains.

In an attempt to throw some light on this question the relative activities of homobiotin for 8 different strains of staphylococci were tested. As shown 
in Table 5, the quantity of this homolog required for half-maximum growth was relatively constant among the individual strains. Other than cultures H3A and $\mathrm{H} 2 \mathrm{C}$ (these were run in erlenmeyer flasks) the determinations were made in the $18 \mathrm{~mm}$. test tube cultures as previously described. Under these conditions precise estimation for half-maximum growth could not be made and it is believed that the variations cited, other than culture $\mathrm{H} 10 \mathrm{~A}$, could be laid to experimental error. Culture H10A also required unusually high amounts of biotin, thus indicating that no significance could be attached to its high homobiotin requirements. This culture will be discussed more thoroughly in a different section.

These results are rather difficult to interpret. If homobiotin were first converted to biotin before being utilized, the rates of conversion might be expected to differ widely among different strains and thus be reflected in different activities among the cultures. Among the cultures tested this was not the case. On the other hund, a constancy in activity of the homolog as was apparently obtained might merely indicate a small contamination of biotin in the homobiotin, itself. Cultures $\mathrm{H} 3 \mathrm{~A}$ and D2A required .03-.05 millimicrograms of biotin per $10 \mathrm{ml}$. for half-maximum growth, thus indicating that biotin, itself, was 1000-1500 times as active as homobiotin. A contamination of the homolog with less than 0.1 per cent biotin could then explain the results obtained. On the other hand, an antibiotin effect of homobiotin, as illustrated in Table 4, could not be demonstrated if it were contaminated with biotin. It is therefore tentatively concluded that these organisms are using homobiotin as such in their metabolism without converting it into biotin.

Biocytin: - WRIGHT et al. (1950a) reported the isolation in crystalline form of a component of yeast extract, termed biocytin, that was available as a source of biotin to Lactobacillus casei but not to Lactobacillus arabinosus. Acid or alkaline hydrolysis yielded free biotin. Further characterization of this substance (WRIGHT et al. [1950b]) revealed that it served as a source of biotin for a number of different microorganisms; that it was stable to a number of commercial enzyme preparations; that it was combinable with avidin, and, that it was relatively inactive as compared to biotin in reversing the bacteriostatic activity of certain biotin antimetabolites. The purified crystalline substance con tains about 65 per cent biotin.

Although biocytin has not been described as having antibiotin properties for any microorganisms tested, it was thought desirable to test the biotin activity 
of this substance upon the staphylococci used in this study. As shown in Table 3 , biocytin in the concentration of 4 millimicrograms per $10 \mathrm{ml}$. effectively replaced the biotin requirements for the 18 strains tested. Table 6 demonstrates that on a weight basis biotin is from 1.4 to 2.0 times as ative as biocytin for the three strains tested. It would, therefore, seem, that within the limits of error biotin and biocytin have equal activities for these microorganisms when calculated on a molar basis.

Mention has been made that culture H10A acquired unusually high concentrations of biotin for growth. This is illustrated in Table 3 wherein it is shown that neither $2 \mathrm{~m} \mu \mathrm{g}$. of biotin, or $4 \mathrm{~m} \mu \mathrm{g}$. of biocytin per $10 \mathrm{ml}$. of medium were sufficient to influence growth. Of additional interest is the fact that the culture appears to have limited abilities to synthesize biotin. In repeated experiments, including serial subculture, this organism always grew to a slight extent in the basal medium. This small amount of growth could not have resulted from biotin contamination in the basal medium. This is amply demonstrated by the fact that this organism required at least a 100 -fold quantity of biotin for half-maximum growth as did cultures H3A and D2A. These results are presented in Table 6. Accordingly, a similar increase in biocytin concentration was necessary for half-maximum growth. Table 5 also illustrates the high requirement for homobiotin for this organism.

These results would indicate that this culture has an unusually high demand for biotin in its metabolism. Further study of this culture may prove to be fruitful.

Oleic acid: - During the past few years it has hecome generally accepted that a combination of oleic acid and aspartic acid will substitute for biotin for those organisms that require this vitamin. However, oleic acid is, itself, very toxic for most microorganisms, and the range of concentration that can be added to a medium without encountering growth inhibition is very narrow. In order to avoid the toxic effects of oleic acid, it is usually added to the medium in the form of Tween 80 . This substance (polyoxyethylene sorbitan mono-oleate) satisfies the oleic acid requirements of various microorganisms tested but does not display in toxicity. 
Although aside from the general problem at hand, an attempt was made to determine whether the coagulase-negative staphylococci would also grow in a biotin free medium when supplied with Tween 80. As shown in Table 3, all but one strain grew satisfactorily in such a medium. Strain S36 was unable to grow in this medium. Even after incubation for six days growth in the medium was no better than that occurring in the control. A further study concerning the relalionship of oleic acid and biotin in the nutrition of this culture would seem to be warranted. Lack of time did not permit this study.

\section{I) Iscussiton}

No attempt was made to incorporate any of the ahove-mentioned substances into a selective of differential plating medium for the purpose of detecting the coagulase-positive staphylococci in foods and other materials. These studies would indicate that each of the antibiotin substances tested would be of little or no value in inhibiting the coagulase-negative strains in such a medium. It should be realized, however, that the substances having anti-biotin properties were far from exhausted in this study, and that one may yet be found that will be' of value in achieving the goal sought.

In spite of the failure to achieve the practical results desired, it would seem that this study has divulged a number of peculiarities in the biotin metabolism of a few of the Staphylococcus strains. Further study of these apparent anomalies might be of value in contributing to our general knowledge of bacterial physio$\log$.

\section{SUMMARY}

The biotin activity, or anti-biotin properties of a number of substances, was tested on a group of biotin-requiring, coagulase-negative Staphylococcus strains. Of the 18 cultures tested desthiobiotin served as a source of biotin for 17 strains. This substance appeared to be 40 per cent as active as biotin in supporting growth for two of the cultures tested. This substance possessed antibiotin properties for one strain (S19).

Homohiotin, one of the chemical homologs of biotin. served as a biotin source for 17 of the 18 strains. Its activity appeared to be less than 0.1 per 
cent of that of biotin among the 8 strains tested. Although it replaced biotin in high concentrations, small quantities appeared to possess anti-biotin properties for one of the Staphylococcus strains. Homobiotin acted as an anti-biotin sub. stance for strain S36 in alj concentrations tested.

In contrast to studies reported on other biotin-requiring microorganisms, there appeared to be no stoichiometric relationship between biotin and avidin in the nutrition of Staphylococcus, strain H17C. Upon adding increasing increments of egg white to a medium containing a limited amount of biotion, growth was unaffected until a critical concentration of avidin was reached. At this level little or no growth of the microorganism occurred. At higher levels growth again occurred, the amount depending directly upon the level of egg white added.

All Staphylococcus cultures tested (18 strains) were able to utilize biocytin in place of biotin. On a molar basis this substance appeared to have an activity equal to that of biotin.

Strain S36 failed to grow in a biotin deficient medium to which 0.1 per cent Tween 80 was added. All other strains tested were able to grow under these conditions.

In comparison with other strains culture H10A appeared to require ap. proximately 100 times as much biotin for growth. This high requirement could be demonstrated in spite of the fact that the culture was able to grow to a limited degree in the absence of added biotin. This culture also demonstrated similar high requirements for biocytin, homobiotion, and desthiobiotin in a biotin free medium.

\section{ACKNOWLADGEMEN'S}

The authors wish to thank Dr. S. H. Rubin, of Hoffmann.La Roche, Inc., Nutley, New Jersey, for the homobiotin used in this study. Appreciation is also expressed to Dr. L. D. Wright, of Sharpe \& Dohme, Inc., Glenolden, Pennsylvania, for the generous sample of biocytin. 
TABIA 1

Basal Medium

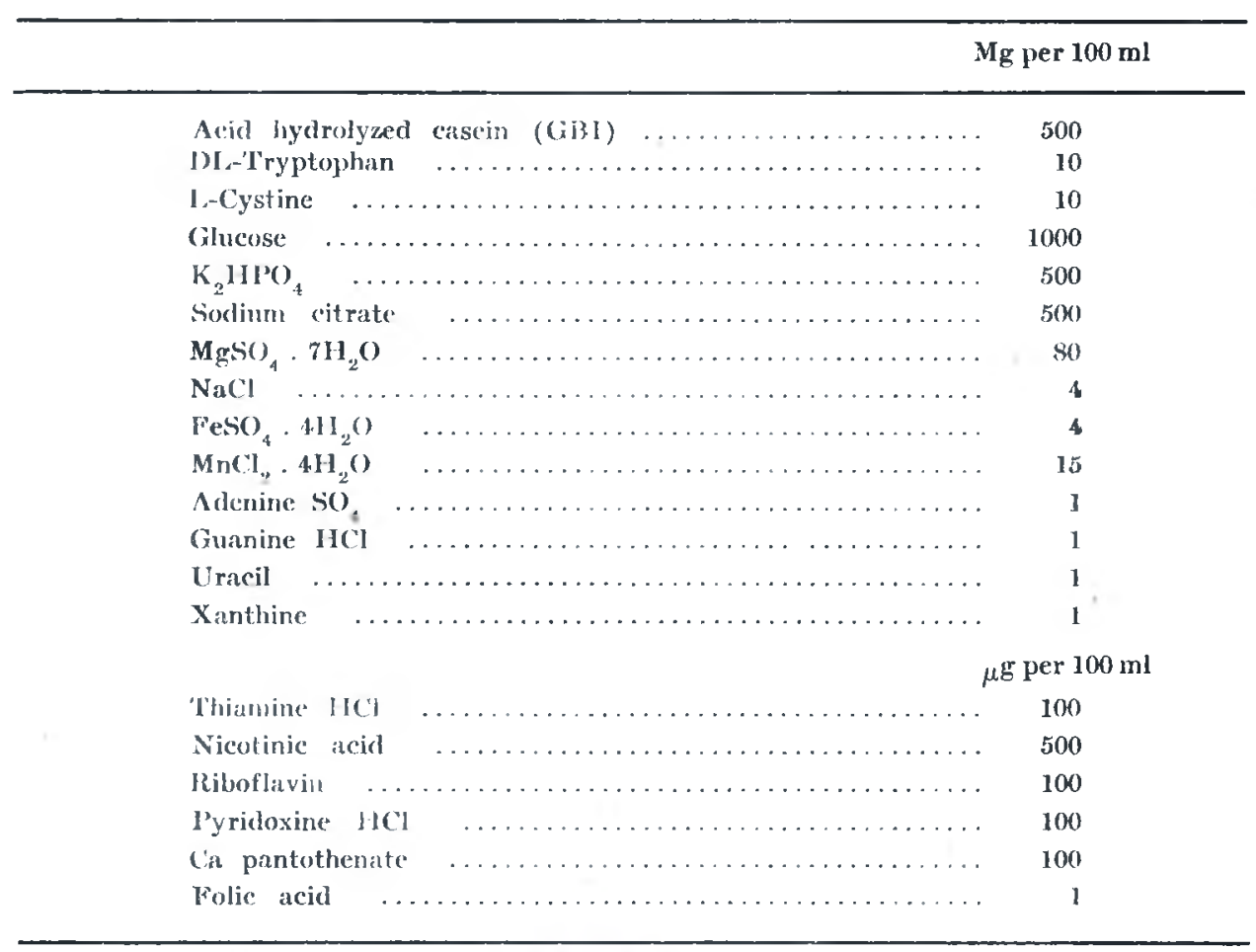

'TABI,E: 2

Effect of Different Concentrations of Raw Egg White upon the Growth of staphylococcus sp. H17C in a Medium Containing $2 \mathrm{~m}_{\mathrm{g}} \mathrm{g}$ Biotin per $10 \mathrm{ml}$.

\begin{tabular}{c|c}
$\begin{array}{c}\text { Raw egg white } \\
\text { (per cent) }\end{array}$ & $\begin{array}{c}\text { Growth, 47 hrs. } \\
\text { (optical density x 100) }\end{array}$ \\
\hline 0 & 130 \\
.02 & 110 \\
.04 & 110 \\
.06 & 110 \\
.08 & 110 \\
.10 & 110 \\
.15 & 36 \\
.20 & 23 \\
.25 & 37 \\
.30 & 52 \\
.40 & 51 \\
.50 & 62 \\
1.0 & 62 \\
5.0 & 110 \\
\hline
\end{tabular}


TABLE 3

Growth Kesponse of Coagulase-Negative Staphylococcus Strains 'Toward Biotin and Related Substances. Readings are Recorded in Terms of Optical Density $x 100$.

\begin{tabular}{|c|c|c|c|c|c|c|c|c|}
\hline & 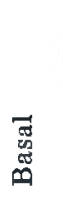 & 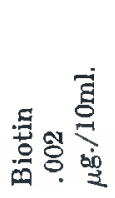 & 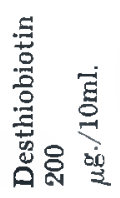 & 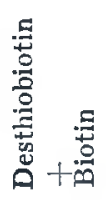 & 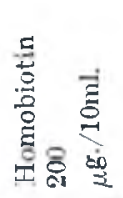 & 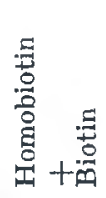 & 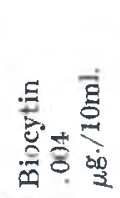 & 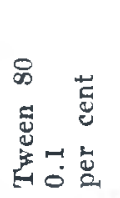 \\
\hline H3A & 4 & 24 & - & - & - & - & 39 & 48 \\
\hline H12A & 19 & 48 & 68 & 56 & 60 & 62 & 55 & 54 \\
\hline D2A & 1 & 42 & 34 & 415 & 4.8 & 45 & 48 & 28 \\
\hline H9A & 23 & 51 & 62 & 71 & 88 & 53 & 53 & 43 \\
\hline H8A & 43 & 88 & 90 & 94 & 92 & 88 & 98 & 90 \\
\hline $20-10$ & 23 & 90 & 58 & 63 & 75 & 95 & 7.5 & 120 \\
\hline H13A & 7 & 37 & 33 & 42 & 45 & 37 & 35 & 55 \\
\hline S19 & 27 & 75 & 0 & 83 & 86 & 79 & 72 & 65 \\
\hline $\mathrm{H} 2 \mathrm{C}$ & 7 & 43 & 28 & 47 & 50 & 44 & 50 & 48 \\
\hline 4 & 25 & 90 & 95 & 100 & 90 & $9: 3$ & 100 & 90 \\
\hline DIA & 17 & 68 & 66 & 67 & 75 & 70 & 45 & 51 \\
\hline S36 & 4 & 60 & 57 & 60 & 0 & 0 & 60 & (6 \\
\hline $\mathrm{H} 17 \mathrm{C}$ & 12 & 110 & 110 & 110 & 110 & 120 & 120 & 120 \\
\hline F1A & 17 & 50 & 55 & 60 & 62 & 62 & 49 & 69 \\
\hline $\mathrm{H7C}$ & 5 & 58 & 32 & 52 & 73 & 62 & 35 & $4 !$ \\
\hline S9 & 22 & 84 & 86 & 86 & 86 & 86 & 94 & 85 \\
\hline H11B & 10 & 45 & 32 & 52 & 47 & 54 & 54 & 62 \\
\hline H10A & 29 & 27 & 62 & 48 & 71 & 63 & 27 & - \\
\hline H10B & 37 & 4 & 75 & 75 & 80 & 72 & $10 \mathrm{C}$ & 57 \\
\hline
\end{tabular}

TABLE 4

Effect of Homobiotin upon the Growth of Staphylococcus sp. H17C.

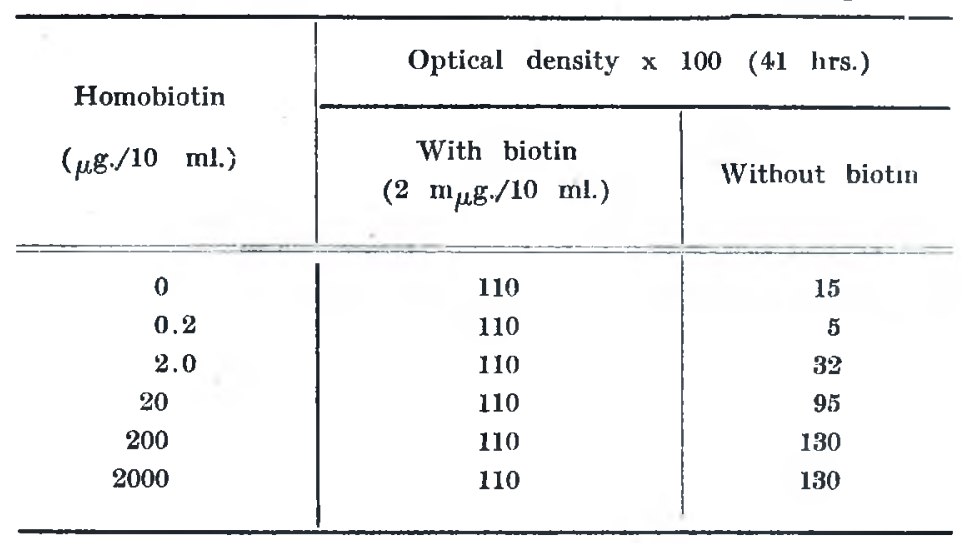


TABLE 5

Comparative Activity of Homobiotin for

Different Staphylococous Strains.

\begin{tabular}{l|c}
\hline & $\begin{array}{c}\text { Homobiotin necessary for } \\
\text { Culture }\end{array}$ \\
& $\begin{array}{c}\text { maximum growt } \\
(\mu \mathrm{g} . / 10 \mathrm{ml})\end{array}$ \\
\hline H9A & 1 \\
H13A & $0.3-1$ \\
D2A & 0.3 \\
H17C & $1-3$ \\
S19 & $0.3-1$ \\
H3.A & $0.4-0.8$ \\
H2C & 0.4 \\
H10A* & $10-30$ \\
\hline
\end{tabular}

- Culture H10A also requires unusually large amounts of biotin for $1 / 2$ maximum growth.

\section{TABLE 6}

Comparative Activities of Biotin and Biocytin.

\begin{tabular}{l|c|c|c}
\hline \multirow{2}{*}{ Culture } & \multicolumn{2}{|c|}{$\begin{array}{c}\text { Quantities required for 1/2 } \\
\text { max. growth }\end{array}$} & \multirow{2}{*}{$\begin{array}{c}\text { Biocytin } \\
\text { Biotin }\end{array}$} \\
\cline { 2 - 3 } & Biotin & Biocytin & \\
\hline \hline H3A & $.03-.05$ & $.05-0.1$ & 1.9 \\
D2A & .05 & 0.1 & 2.0 \\
H10A & $5-10$ & $10-30$ & 1.4 \\
\hline
\end{tabular}




\section{REFERENCES}

Bhlcher, M. Rurf, and Licistis, Hermas C. - 1949 - Girowth promotion and antibiotin effect of homobiotin and norbiotin. J. Bact., 58, 579-583.

Evans, J. B. - 1948 - Studies of staphylococci with special reference to the coagulasepositive types. J. Bact., 55, 793-800.

Evans, James B., and Niren, C. F. Jr. - $1950-$ A comparative study of known foodpoisoning staphylococci and related varieties. J. Bact., 59, 545-550.

Evans, James B., BuetTnem, I. G., and Niven, C. F., JR. … 1950 - Evaluation of coagulase test in the study of staphylococei associated with food poisoning. J. Bact., 60, 481-484.

Gornherg, M. W., Sternach, L. H., Kaiser, S., Heineman, S. D., Sicheiner, J., and Rumin, S. H. - 1947 - Antibiotin effect of some biotin homologs. Arch. Biochemistry, 14, 480-482.

Gnertiz, Albert C. - 1950 - A study of the vitamin nutrition of staphylococci with special reference to biotin requirements. Thesis, Department of Bacteriology and Parásitology. University of Chicago. December, 1950.

Gretrlen, Ajbert C., and Evans, James B. - 1951 - Vitamin requirements of staphylococci. Bact. Proc., Soc. Am. Bact., 1951.

Hutwer, S. H., and Bjerknes, C. A. - 1948 - Volatile preservatives for culture media. Proc. Soc. Exptl. Biol. Med., 67, 393-39\%.

Runin, S. H., Drexter, L., and Mayer, E. H. - 1945 - Biological activity of synthetic d,1-desthiobiotin. Proc. Soc. Exptl. Biol. Med., 58, 352-356.

Rubis, SaUl. H., and Scheiner, Jacon - 1949 - Antibiotic effect of homologs of biotin and biotin sulfone. Arch. Biochemistry, 23, 400-410.

Srokes, Jacor L., and Gun ness, Marion - 1945 - Microbiological activity of synthetic biotin, its optical isomers, and related compounds. J. Biol. Chem., 15\%, 121-126.

Wright, L. D., Cresson, E. L., Skegg, H. R., Wood, 'T. R., Peck, R. L., Wolf, D. E., and Folkens, K. - 1950a - Biocytin, a naturally-occurring complex of biotin. J. Am. Chem. Soc., 72, 1048.

Wright, Lemuex D., Chesson, Emelox L., Valentick, Katimenine and Skeggs, Heisk R. - 1950b - Microbiological characterization of biocytin. Fed. Proc., 9, 374. 